\title{
In-silico investigation of bitter hop-derived compounds and their cognate bitter taste receptors
}

Andreas Dunkel, ${ }^{\dagger}$ Thomas Hofmann ${ }^{\ddagger}$ and Antonella Di Pizio, ${ }^{*}, \dagger$

tLeibniz-Institute for Food Systems Biology at the Technical University of Munich, Lise-Meitner Str. 34, D-85354 Freising, Germany, ${ }^{\ddagger}$ Chair of Food Chemistry and Molecular Sensory Science, Technical University of Munich, Lise-Meitner-Straße 34, D-85354 Freising, Germany,

corresponding authors

*E-MAIL a.dipizio.leibniz-Isb@tum.de 
Figure S1. 2D representation of the interactions between isohumulones 1-6 and TAS2R1 binding site residues

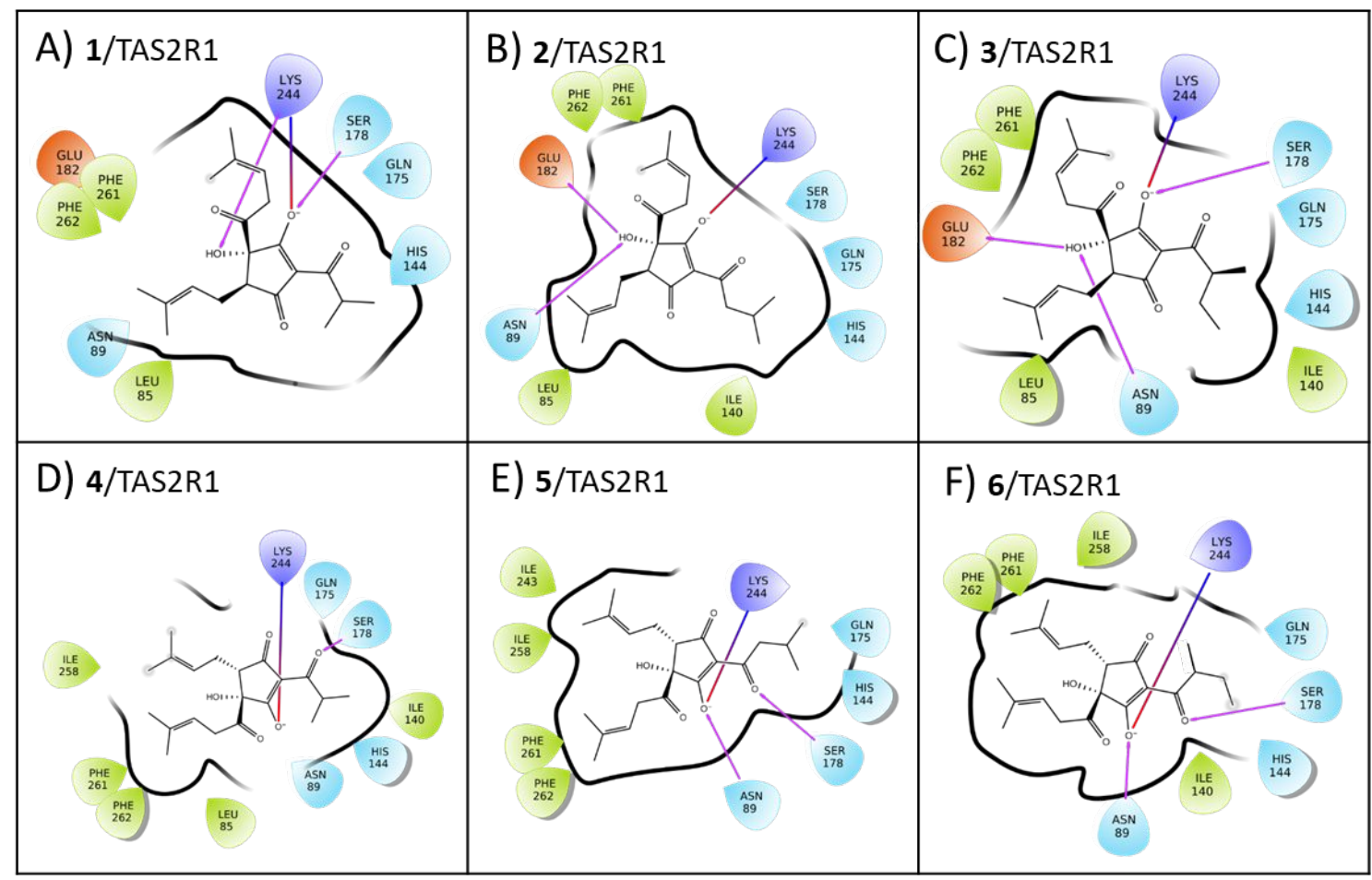

Figure S2. 2D representation of the interactions between isohumulones 1-6 and TAS2R14 binding site residues

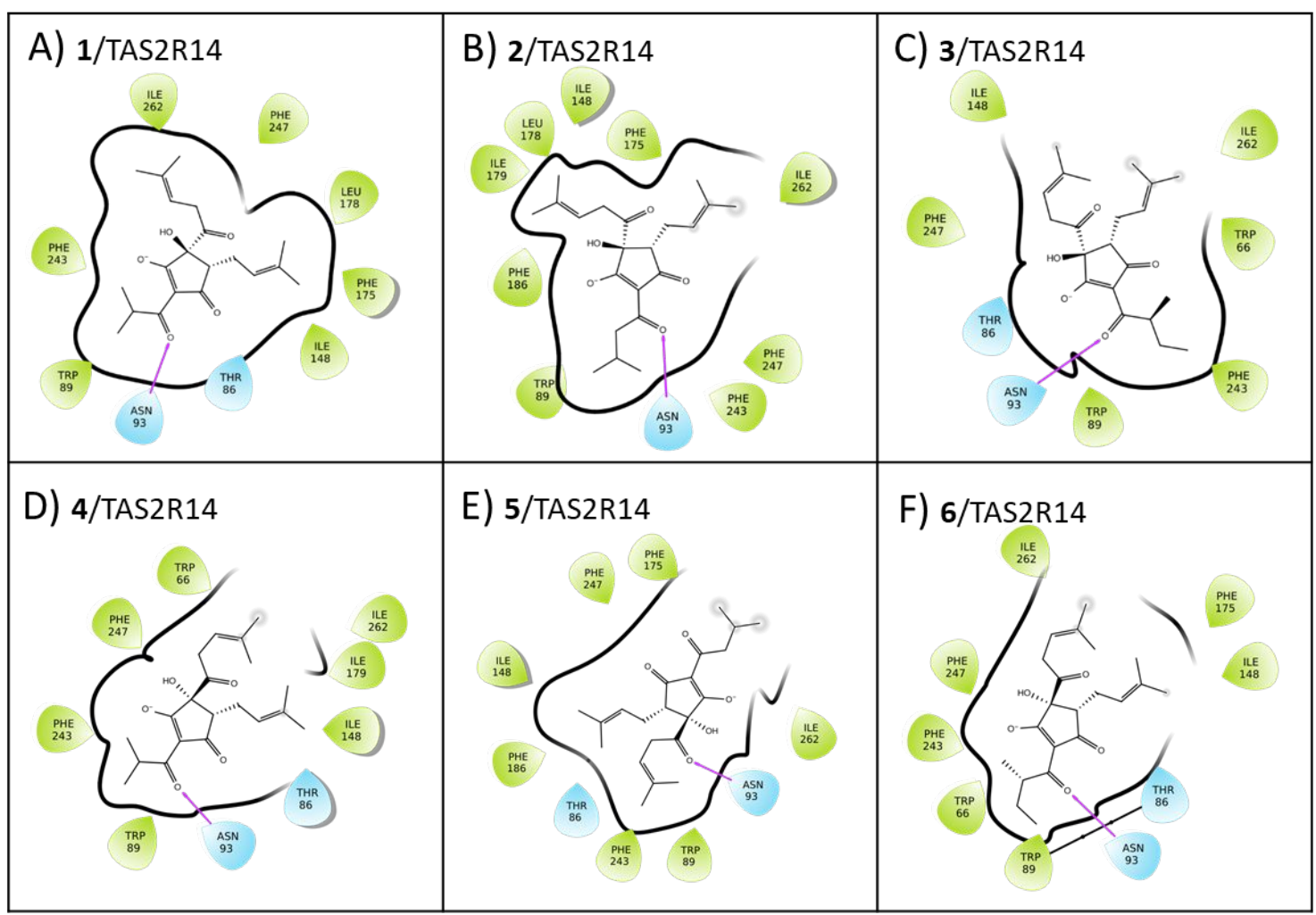


Figure S3. 2D representation of the interactions between compounds 7-9 and TAS2R1 binding site residues

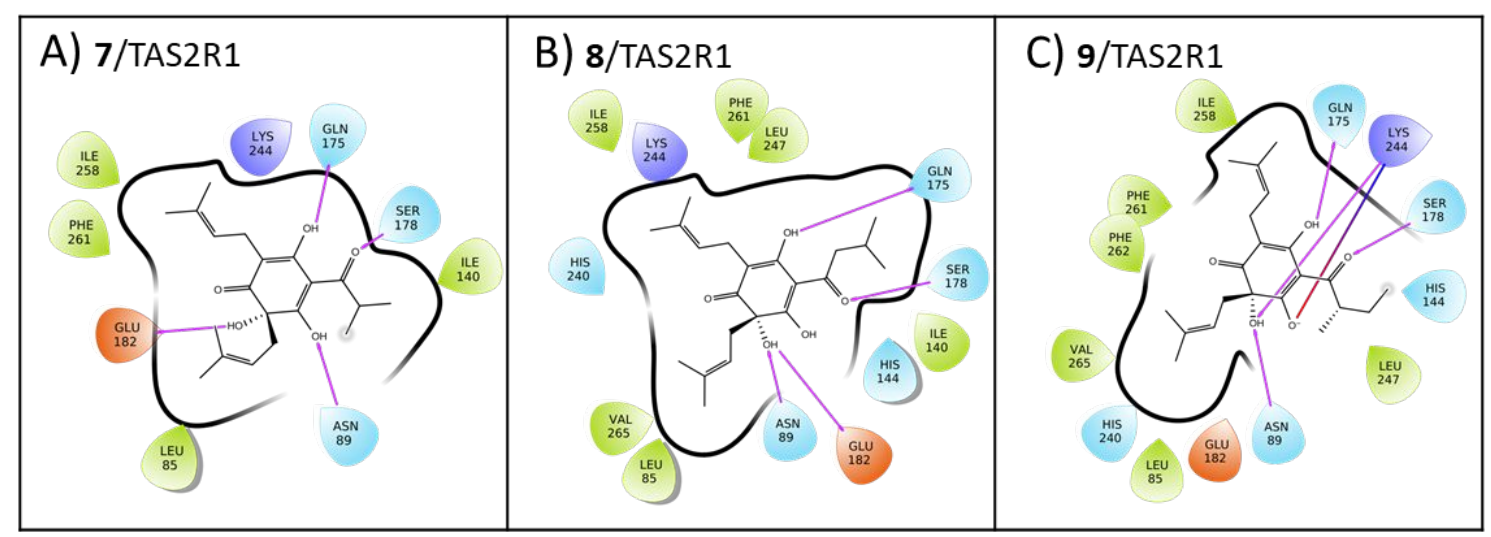

Figure S4. 2D representation of the interactions between compounds 7-9 and TAS2R40 binding site

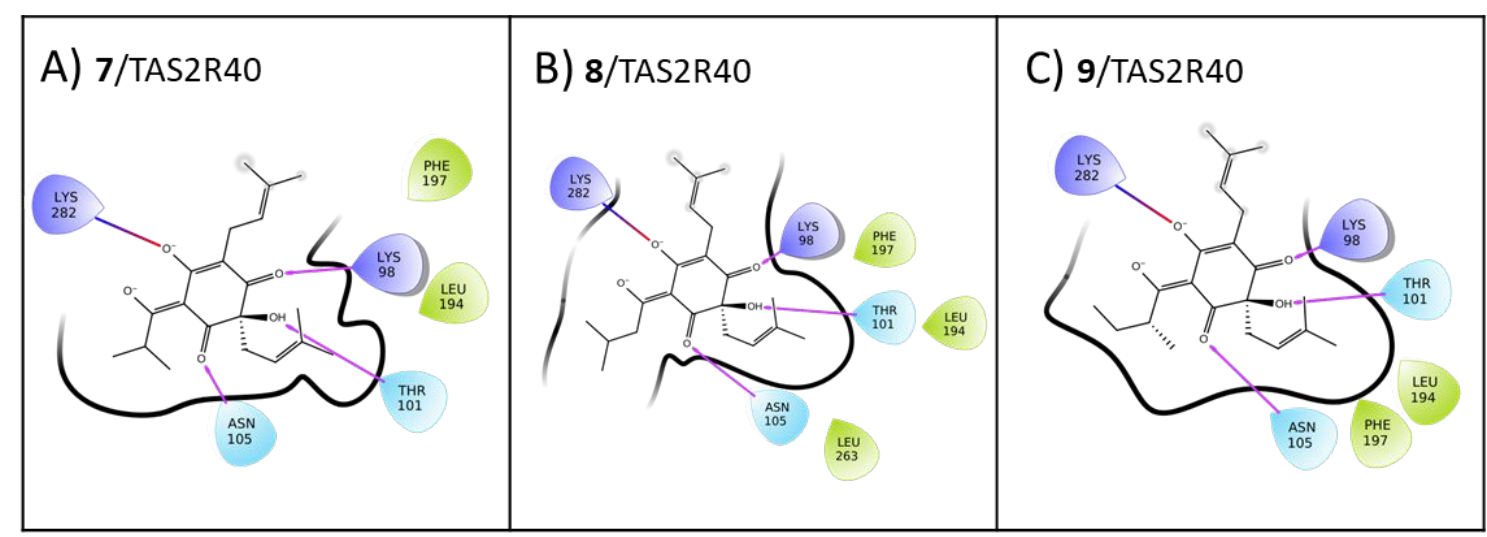

Figure S5. 2D representation of the interactions between compound 11 and TAS2R40 binding site

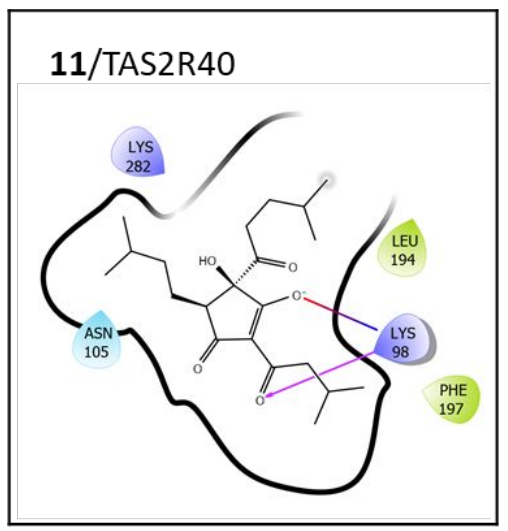


Figure S6. 2-dimensional t-SNE plot of the bitter chemical space, coloured according FooDB and DrugBank occurrence (t-SNE 2 and 3)

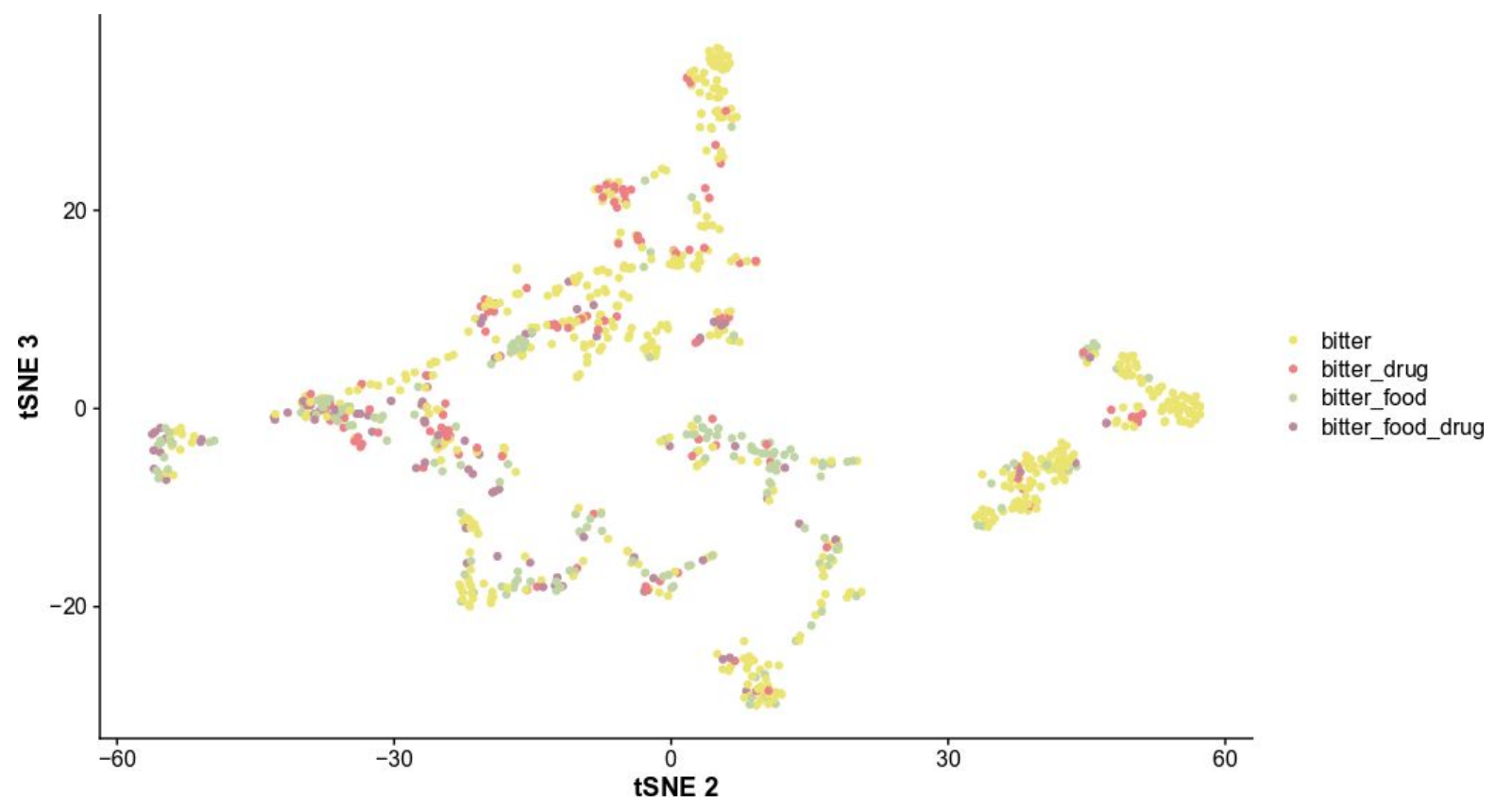

Figure S7. 2-dimensional t-SNE plot of the bitter chemical space, coloured according to ClassyFire chemical superclasses (t-SNE 2 and 3 )

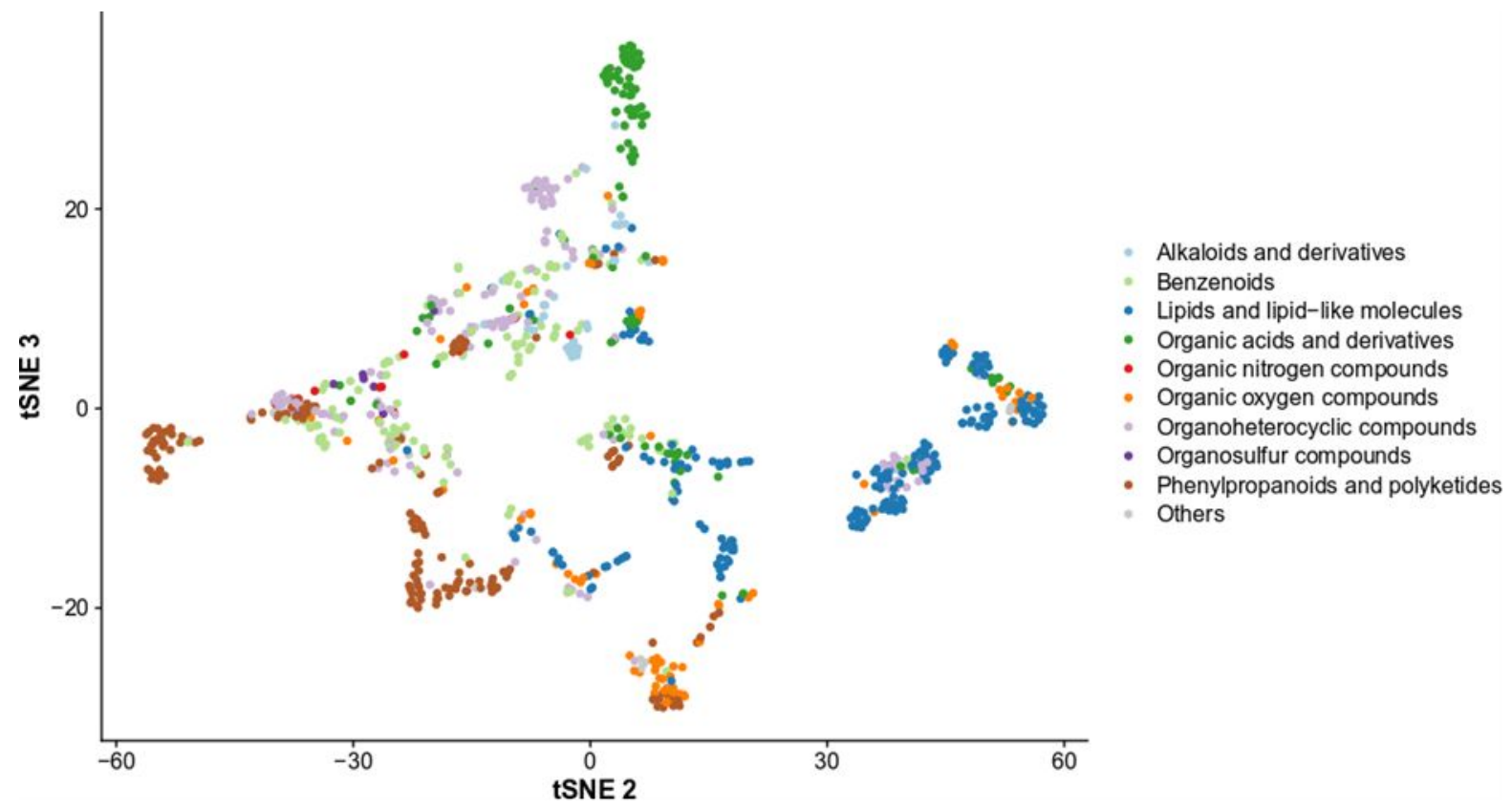


Figure S8. 2-dimensional t-SNE plot of the bitter (in yellow) chemical space, hop-derived compounds 1-11 are coloured in black (t-SNE 2 and 3)

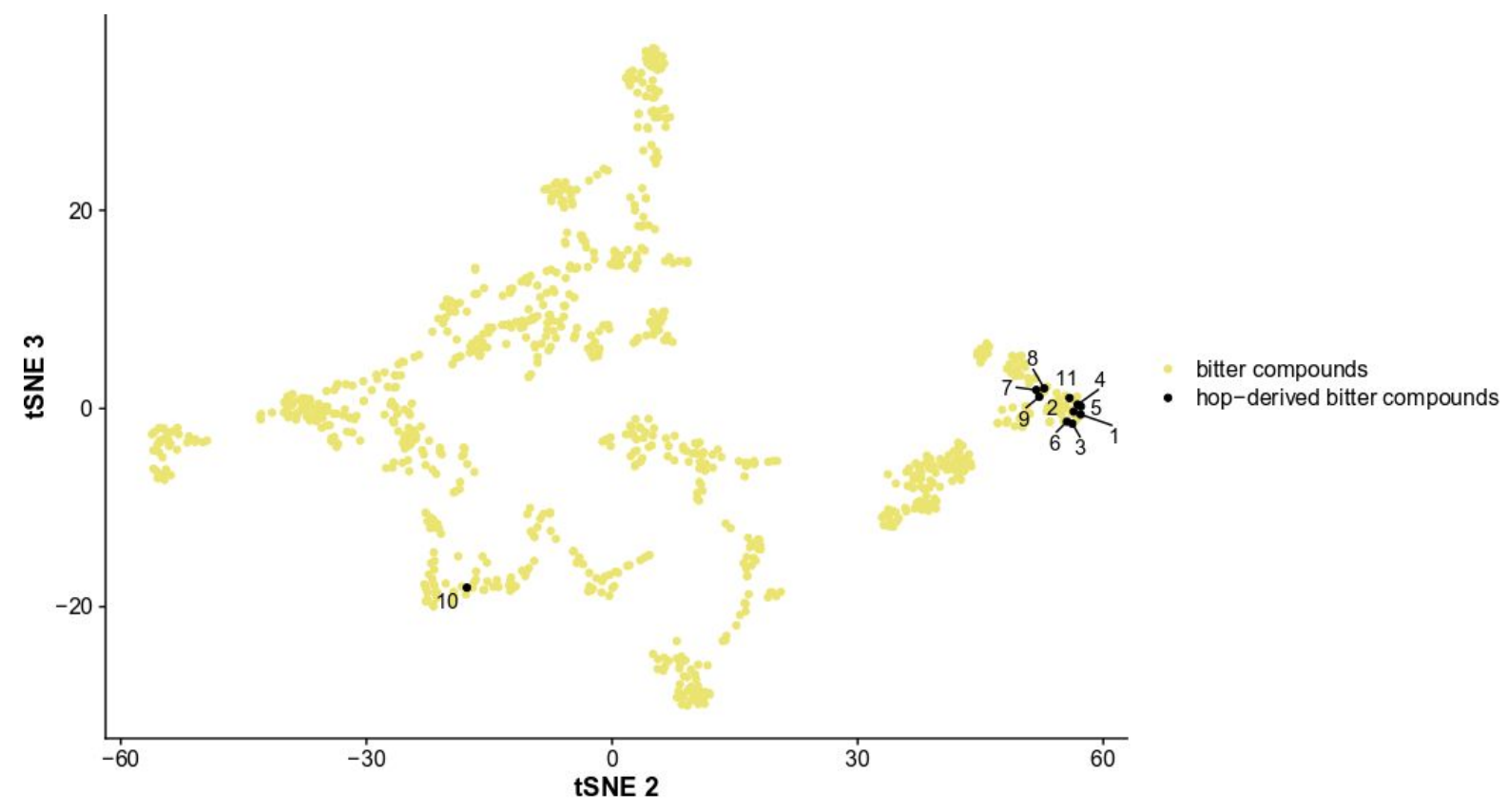


Table S1. Number $(\mathrm{N})$ of BitterDB compounds for each ClassyFire chemical superclass

\begin{tabular}{|l|l}
\hline Superclass & N. \\
\hline Alkaloids and derivatives & 32 \\
\hline Benzenoids & 136 \\
\hline Homogeneous non-metal compounds & 1 \\
\hline Hydrocarbon derivatives & 1 \\
\hline Hydrocarbons & 1 \\
\hline Lignans, neolignans and related compounds & 3 \\
\hline Lipids and lipid-like molecules & 208 \\
\hline Nucleosides, nucleotides, and analogues & 2 \\
\hline Organic acids and derivatives & 108 \\
\hline Organic nitrogen compounds & 11 \\
\hline Organic oxygen compounds & 73 \\
\hline Organic Polymers & 1 \\
\hline Organohalogen compounds & 1 \\
\hline Organoheterocyclic compounds & 143 \\
\hline Organosulfur compounds & 8 \\
\hline Phenylpropanoids and polyketides & 159 \\
\hline NA & 4 \\
\hline
\end{tabular}


Table S2. Number $(\mathrm{N})$ of BitterDB compounds for each ClassyFire chemical class

\begin{tabular}{|c|c|}
\hline Class & $\mathbf{N}$. \\
\hline Anthracenes & 1 \\
\hline Aurone flavonoids & 1 \\
\hline Azepines & 2 \\
\hline Azoles & 6 \\
\hline Azolidines & 3 \\
\hline Azolines & 1 \\
\hline Benzene and substituted derivatives & 105 \\
\hline Benzimidazoles & 2 \\
\hline Benzodiazepines & 2 \\
\hline Benzodioxoles & 3 \\
\hline Benzofurans & 2 \\
\hline Benzopyrans & 7 \\
\hline Benzothiazepines & 1 \\
\hline Benzothiazines & 1 \\
\hline Benzothiazoles & 6 \\
\hline Benzoxepines & 1 \\
\hline Carboximidic acids and derivatives & 2 \\
\hline Carboxylic acids and derivatives & 91 \\
\hline Cinchona alkaloids & 7 \\
\hline Cinnamic acids and derivatives & 22 \\
\hline Cinnamyl alcohols & 1 \\
\hline Coumarins and derivatives & 11 \\
\hline Depsides and depsidones & 3 \\
\hline Diazanaphthalenes & 2 \\
\hline Diazinanes & 1 \\
\hline Diazines & 25 \\
\hline Dioxolanes & 1 \\
\hline
\end{tabular}




\begin{tabular}{|c|c|}
\hline Dioxolopyrans & 1 \\
\hline Emetine alkaloids & 3 \\
\hline Ergoline and derivatives & 1 \\
\hline Fatty Acyls & 41 \\
\hline Flavonoids & 71 \\
\hline Flavonolignans & 1 \\
\hline Furopyrans & 3 \\
\hline Glycerolipids & 8 \\
\hline Halohydrins & 1 \\
\hline Harmala alkaloids & 1 \\
\hline Heteroaromatic compounds & 2 \\
\hline Hydroxy acids and derivatives & 1 \\
\hline Imidazopyrimidines & 9 \\
\hline Indoles and derivatives & 3 \\
\hline Isocoumarans & 1 \\
\hline Isoflavonoids & 18 \\
\hline Isoquinolines and derivatives & 2 \\
\hline Isothiocyanates & 1 \\
\hline Keto acids and derivatives & 1 \\
\hline Lactams & 2 \\
\hline Lactones & 7 \\
\hline Linear 1,3-diarylpropanoids & 24 \\
\hline Lupin alkaloids & 7 \\
\hline Macrolides and analogues & 1 \\
\hline Morphinans & 4 \\
\hline Naphthalenes & 5 \\
\hline Naphthopyrans & 2 \\
\hline Organic carbonic acids and derivatives & $s 2$ \\
\hline Organic sulfonic acids and derivatives & 2 \\
\hline Organic sulfuric acids and derivatives & 1 \\
\hline
\end{tabular}




\begin{tabular}{|c|c|}
\hline Organonitrogen compounds & 11 \\
\hline Organooxygen compounds & 73 \\
\hline Other non-metal organides & 1 \\
\hline Oxanes & 1 \\
\hline Phenanthrenes and derivatives & 1 \\
\hline Phenol esters & 1 \\
\hline Phenol ethers & 7 \\
\hline Phenols & 14 \\
\hline Phthalide isoquinolines & 1 \\
\hline Piperidines & 7 \\
\hline Polypeptides & 1 \\
\hline Prenol lipids & 118 \\
\hline Pteridines and derivatives & 1 \\
\hline Purine nucleosides & 2 \\
\hline Pyranopyridines & 1 \\
\hline Pyrans & 2 \\
\hline Pyridines and derivatives & 11 \\
\hline Pyridopyrimidines & 1 \\
\hline Pyrrolidines & 1 \\
\hline Quebrachamine alkaloids & 1 \\
\hline Quinolines and derivatives & 16 \\
\hline Quinolizidines & 1 \\
\hline Steroids and steroid derivatives & 41 \\
\hline Stilbenes & 4 \\
\hline Strychnos alkaloids & 3 \\
\hline Sulfoxides & 2 \\
\hline Tannins & 2 \\
\hline Tetracyclines & 1 \\
\hline Tetrahydroisoquinolines & 1 \\
\hline Tetralins & 2 \\
\hline
\end{tabular}




\begin{tabular}{|l|l}
\hline Thienopyridines & 1 \\
\hline Thioacetals & 1 \\
\hline Thiocarboxylic acids and derivatives & 1 \\
\hline Thioethers & 1 \\
\hline Thioureas & 3 \\
\hline Triazolopyrimidines & 1 \\
\hline Tropane alkaloids & 2 \\
\hline Tropones & 1 \\
\hline Unsaturated hydrocarbons & 1 \\
\hline Vinylogous acids & 7 \\
\hline Yohimbine alkaloids & 1 \\
\hline NA & 7 \\
\hline
\end{tabular}

